\title{
Notes from the Field: Establishing Baseline Data for Pressure Ulcers in a Rwandan Intensive Care Unit
}

\author{
Gedeon Mutabazi ${ }^{1}$, Jessie Silver ${ }^{1}$ \\ ${ }^{1}$ University Teaching Hospital of Kigali, Rwanda
}

\section{Background}

Pressure ulcers are a common iatrogenic medical problem. Pressure ulcers increase the morbidity and mortality of hospitalized patients as well as the length and cost of hospitalization. It was observed that patients in the Intensive Care Unit (ICU) of a large, referral hospital in Kigali, Rwanda, often had pressure ulcers, however, no data on the ulcers existed.

\section{Objectives}

The objective of this study was to establish baseline data and compare prevalence, incidence, and severity of pressure ulcers in this ICU before and after educational classes.

\section{Methods}

Educational classes on pressure ulcer assessment and classification were held for all ICU nurses. After training, data collection was instituted and conducted over a three month period. Patients were screened upon admission and daily for the presence of pressure ulcers, number of ulcers, ulcer location and ulcer severity. All information was documented in a database.

\section{Results}

Currently, 41 patient stays have been assessed. Eleven patients $(27 \%)$ arrived with preexisting ulcers, six patients $(15 \%)$ developed new ulcers during their ICU stay. No pre-existing ulcers notably worsened in severity in the ICU, and new onset ulcers showed a maximum severity of Stage 2. The most common location for both preexisting and new ulcers was the buttocks, and the most common severity for both pre-existing and new onset ulcers was also Stage 2.

\section{Conclusion}

So far, pressure ulcer incidence (new occurrence) has been found to be at $15 \%$, and prevalence at $41 \%$. These statistics suggests that the ICU has an average incidence and moderately high prevalence of pressure ulcers. Further data collection is needed to verify these results. In light of resource limitations and short-staffing, these results are quite positive. Once baseline data is established with further study, the ICU will be able to evaluate the efficacy of future nursing practice changes designed to reduce pressure ulcer incidence and severity in the ICU setting.

Key words: pressure ulcer, ICU, quality indicator 